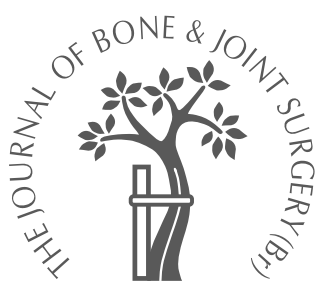

N. Adachi,

M. Ochi,

Y. Uchio,

J. Iwasa,

M. Kuriwaka,

Y. Ito

From Hiroshima

University,

Hiroshima, Japan

\title{
Reconstruction of the anterior cruciate ligament
}

\author{
SINGLE- VERSUS DOUBLE-BUNDLE MULTISTRANDED \\ HAMSTRING TENDONS
}

A total of 108 patients with unilateral instability of the knee, associated with rupture of the anterior cruciate ligament, was prospectively randomised for arthroscopic single- or double-bundle reconstruction of the ligament using hamstring tendons. The same postoperative rehabilitation protocol was used for all. The patients were followed up for a mean of 32 months (24 to 36 ). We measured the anterior laxity and joint position sense at different angles of flexion of the knee to determine whether both bundles in the doublebundle reconstruction contributed to the stability of the joint and proprioception.

No significant difference was found between the two groups with regard to anterior laxity measured by the KT-2000 arthrometer with the knee at $20^{\circ}$ or $70^{\circ}$ flexion nor with regard to proprioception. A notchplasty was required less often in the double-compared with the single-bundle reconstruction.

We did not find any advantage in a double-bundle as opposed to a single-bundle reconstruction in terms of stability or proprioception.

The normal anterior cruciate ligament (ACL) can be divided into two bundles, anteromedial (AM) and posterolateral (PL). Each appears to function at different angles of flexion of the knee, together providing responsibility for the stability of the joint. ${ }^{1,2}$ Reconstruction of the ACL, using multistranded hamstring tendons, has concentrated mainly on replacement of the AM bundle (single-bundle reconstruction). Recent progress in the technique and instrumentation of arthroscopic knee surgery has allowed reconstruction of the two bundles separately to mimic more closely the normal structure of the ACL using hamstring tendons which function at different angles of flexion as in a double-bundle reconstruction. ${ }^{3-5}$ Although some authors have reported good results using a double-bundle technique, ${ }^{6,7}$ few have investigated whether both reconstructed bundles have functional partial responsibility for the stability of the joint at different angles of flexion of the knee.

The ACL not only acts as the main stabiliser of the knee to control anteroposterior (AP) translation and rotation, but is also a neurosensory organ which contributes to proprioception. ${ }^{8-11}$ It has been reported in animal models, that mechanoreceptors can regenerate within the reconstructed ACL, ${ }^{12-15}$ and in clinical practice, proprioception improves gradually after its reconstruction. ${ }^{16}$ It is therefore proposed that the reconstructed AM and PL bundles make different afferent contributions at various angles of flexion.

In this study, we have compared the clinical outcome of double-bundle ACL reconstruction using hamstring tendons, with single-bundle reconstruction in terms of the functional partial responsibility for stability of the knee and proprioception function at different angles of flexion.

\section{Patients and Methods}

Between July 1998 and May 2000, we carried out a randomised, prospective study to compare the clinical results of single-bundle ACL reconstruction, with those of double-bundle reconstruction (Fig. 1). Sixty-eight patients underwent single-bundle reconstruction ( $\mathrm{S}$ group) and 65 double-bundle reconstruction (D group), using multistranded autologous hamstring tendons. We excluded patients with a history of contralateral injury to the knee or those with severe osteoarthritic changes (jointspace narrowing of more than $50 \%$ in any compartment), or those with ligamentous instability of the knee. We excluded ten patients who could not comply with the follow-up protocol. This left 108 patients in the study of whom 55 were in the $S$ group ( 32 men and 23 women) with a mean age of 29.5 years (14 to 49 ) at the time of surgery. The D group com- 


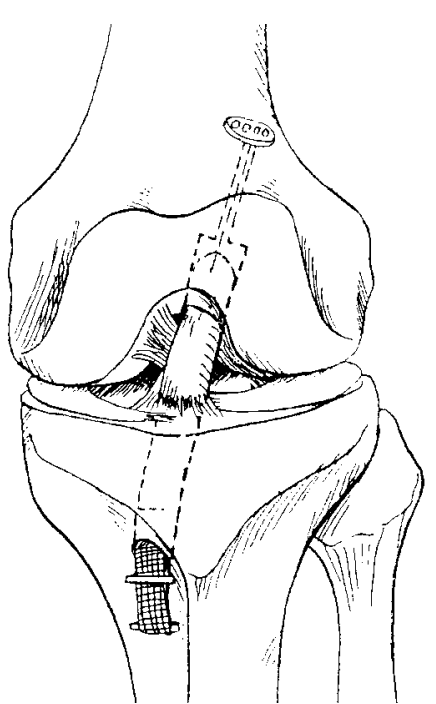

Fig. 1a

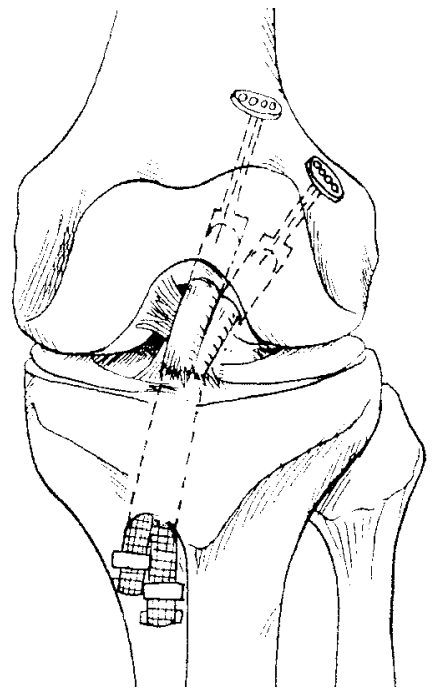

Fig. 1b
Diagrams of a) single-bundle and b) double-bundle reconstruction of the $A C L$.

Table I. Details of the patients in each group

\begin{tabular}{lll}
\hline & S group & D group \\
\hline Mean age (yrs) (range) & 29.5 (14 to 49) & 29.2 (14 to 47$)$ \\
Male:female & $32: 23$ & $33: 20$ \\
Pre-injury activity level (competitive/recreational) & $33 / 22$ & $29 / 24$ \\
Pre-operative anterior laxity (mm) at: & & $6.2 \pm 3.5$ \\
$20^{\circ}$ of flexion & $5.4 \pm 2.9$ & $2.2 \pm 2.2$ \\
$70^{\circ}$ of flexion & $2.7 \pm 1.7$ & 21 \\
Mean interval between injury and operation (mths) & 26 & $10 / 43$ \\
Hamstring tendons used for reconstruction (ST only/ST + G)* & $13 / 42$ & $18 / 4$ \\
Meniscal injury (partial meniscectomy/repair) & $25 / 6$ & 30 \\
Mean follow-up period (mths) & 33 & \\
\hline
\end{tabular}

* ST, semitendinosus; G, gracilis

prised 53 patients ( 33 men and 20 women) with a mean age of 29.2 years (14 to 47 ) at the time of surgery. The details of the patients in each group are given in Table I. All patients had been either competitive or recreational athletes before surgery. The term 'competitive athletes' referred to those who belonged to a sports team in high school, college or beyond, or who regularly participated in organised competition. There were no significant differences in background factors regarding gender, age, pre-injury sports activity, preoperative anterior laxity, time from injury to operation, the quality of tendons used for the reconstruction, meniscal injuries treated by partial meniscectomy or meniscal repair, or the period of follow-up.

The study had ethical approval and informed consent was obtained from all patients.

Operative technique. A skin incision of $4 \mathrm{~cm}$ was made on the medial side of the proximal tibia, over the insertion of the pes anserinus. After incising the expansion of sartorius, the semitendinosus tendon was harvested using a tendon stripper.
For single-bundle reconstruction, we usually quadrupled the semitendinosus tendon to make a bunch of more than 7 $\mathrm{mm}$ in diameter and more than $60 \mathrm{~mm}$ in length. When the tendon was considered to be too thin or too short, the tendon of gracilis was harvested and used in combination with that of semitendinosus to provide a graft of the required dimensions. The mean diameter of the proximal end of the graft as measured by the sizing tubes was $8.2 \pm$ 1.0 (7 to 9). The proximal ends were fixed using an Endobutton CL (Acufex; Smith \& Nephew, Mansfield, Massachusetts), and the distal ends were sutured with Endobutton tape (Acufex; Smith \& Nephew) and fixed with two staples. Only the autologous multistranded tendon spanned the joint space.

For double-bundle reconstruction, if the length of the semitendinosus tendon was greater than $24 \mathrm{~cm}$, the tendon was divided in half. Each tendon was doubled and secured by an Endobutton and Endobutton tape. Thicker grafts were used for the PL bundle and more slender ones for the $\mathrm{AM}$ bundle. If the length of the tendon was less than $24 \mathrm{~cm}$, 


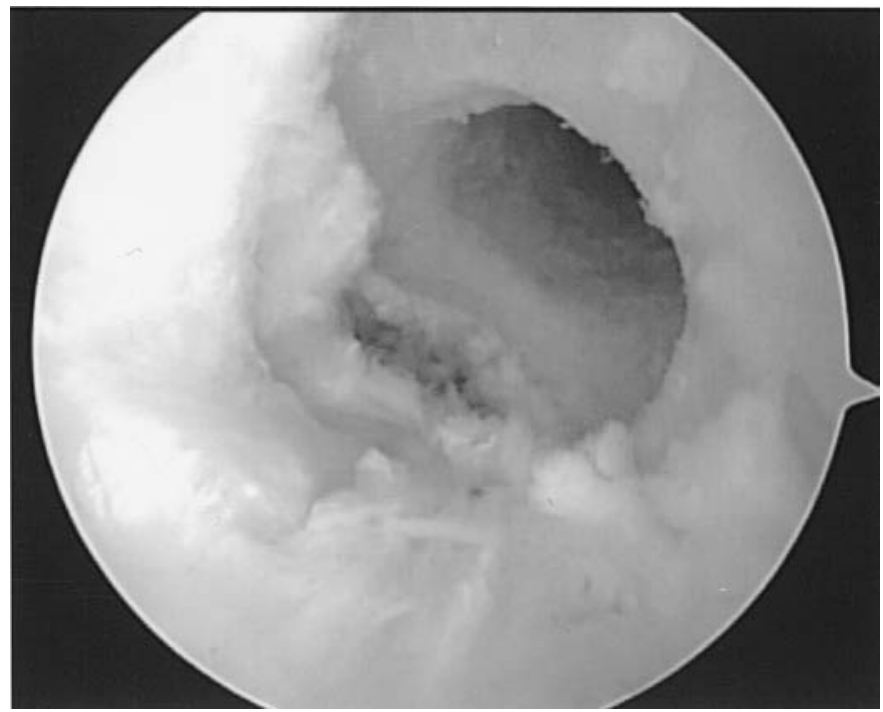

Fig. 2a

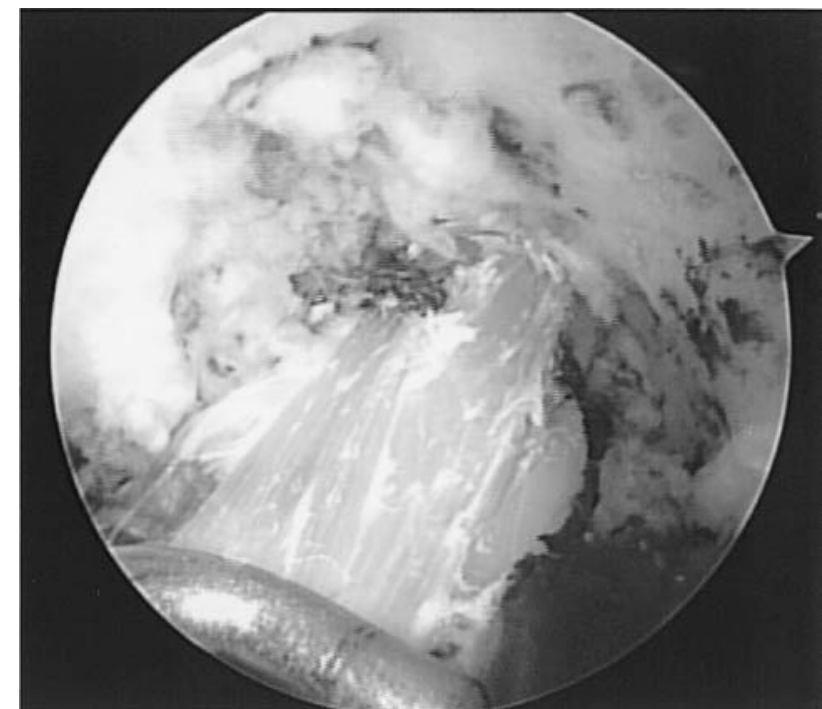

Fig. $2 b$

Arthroscopic views showing the position of the femoral tunnel in a) the single-bundle ACL reconstruction and b) the ACL graft.

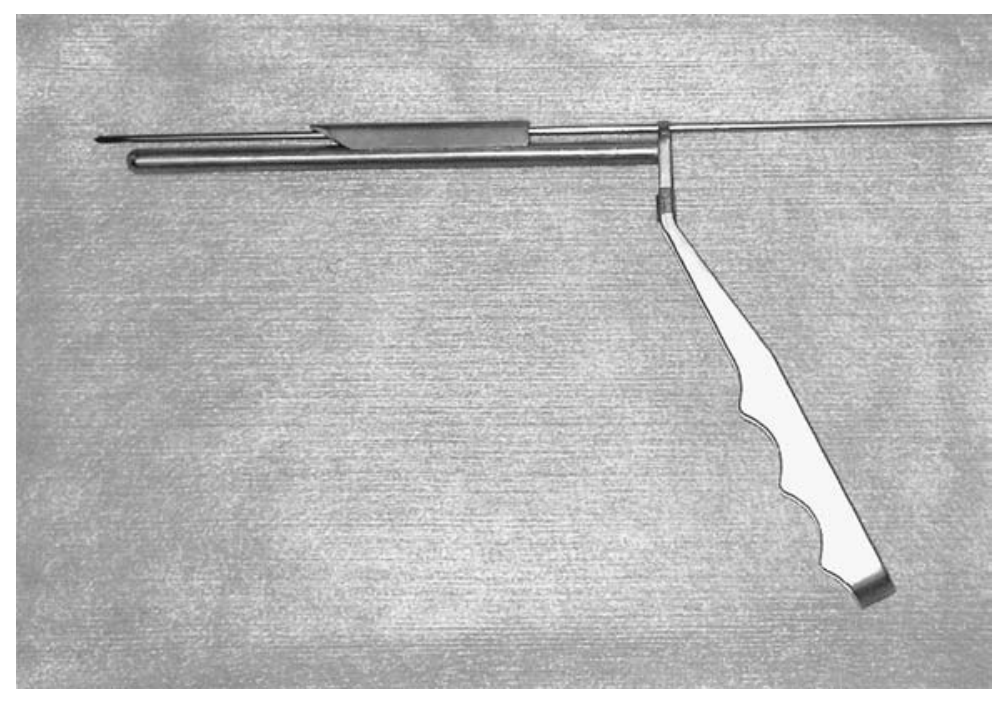

Fig. 3a

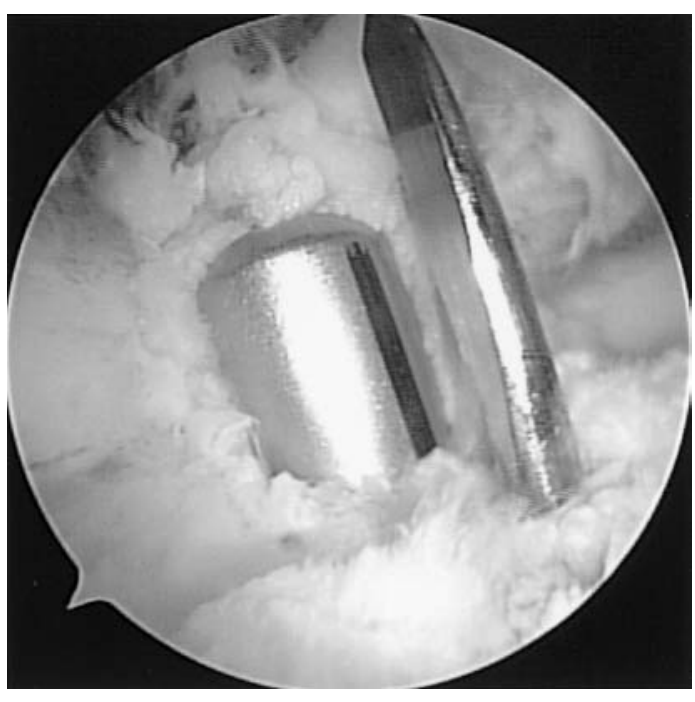

Fig. 3b

Figure $3 a$ - Photograph showing the parallel guide. Figure $3 b$ - Arthroscopic view showing the tip of the K-wire inserted with the parallel guide.

the tendon of gracilis was also harvested. Both the semitendinosus and gracilis tendons were doubled or tripled to make the diameter more than $5 \mathrm{~mm}$. The mean diameter of the proximal end of the PL and AM bundles was $6.8 \pm 1.3$ $\mathrm{mm}(6$ to 7$)$ and $5.9 \pm 1.3 \mathrm{~mm}$ (5 to 7 ), respectively.

$S$ group. Routine arthroscopic inspection was performed through lateral and medial infrapatellar portals with a $30^{\circ}$ oblique arthroscope. The tibial and femoral stumps of the torn ACL were excised using a motorised shaver system. While viewing the tibial attachment of the ACL, a $2.0 \mathrm{~mm}$
Kirschner wire was inserted into the centre of its attachment from the medial aspect of the proximal tibia, using the Pro-trac ACL guide system (Acufex; Smith \& Nephew). With the position of the tip of the K-wire confirmed, we overdrilled the wire with a cannulated reamer of the same diameter as that of the largest diameter of the graft. The position of the femoral tunnel was $5 \mathrm{~mm}$ anterior to the posterior margin of the intercondylar notch and at the 10 o'clock (right) or 2 o'clock (left) position with respect to the apex of the notch (Fig. 2). Through the tibial tunnel, a 2.4 


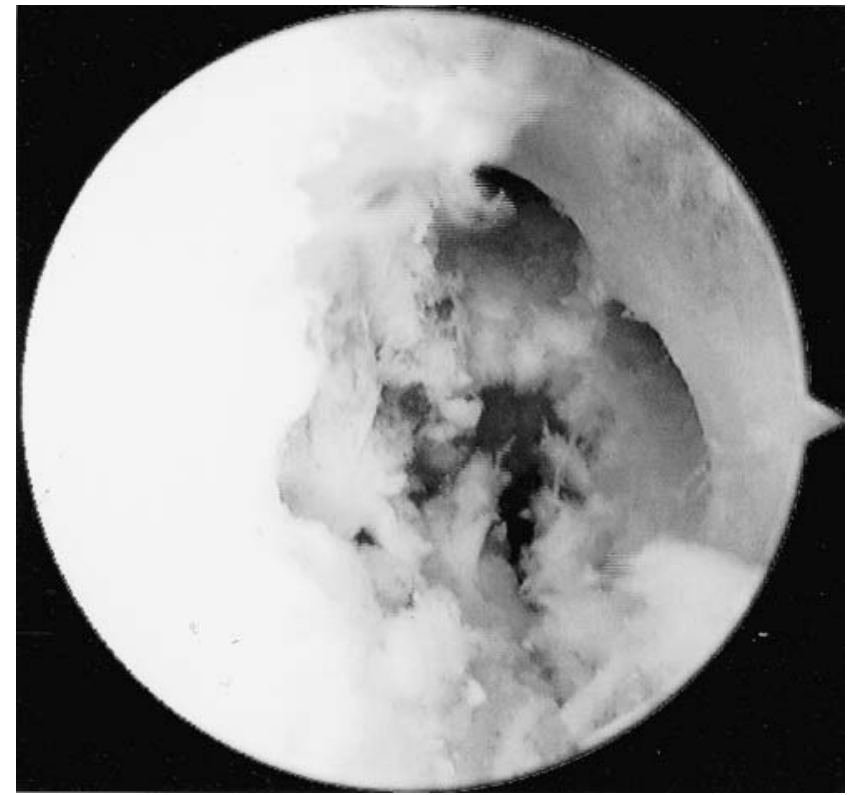

Fig. $4 \mathrm{a}$

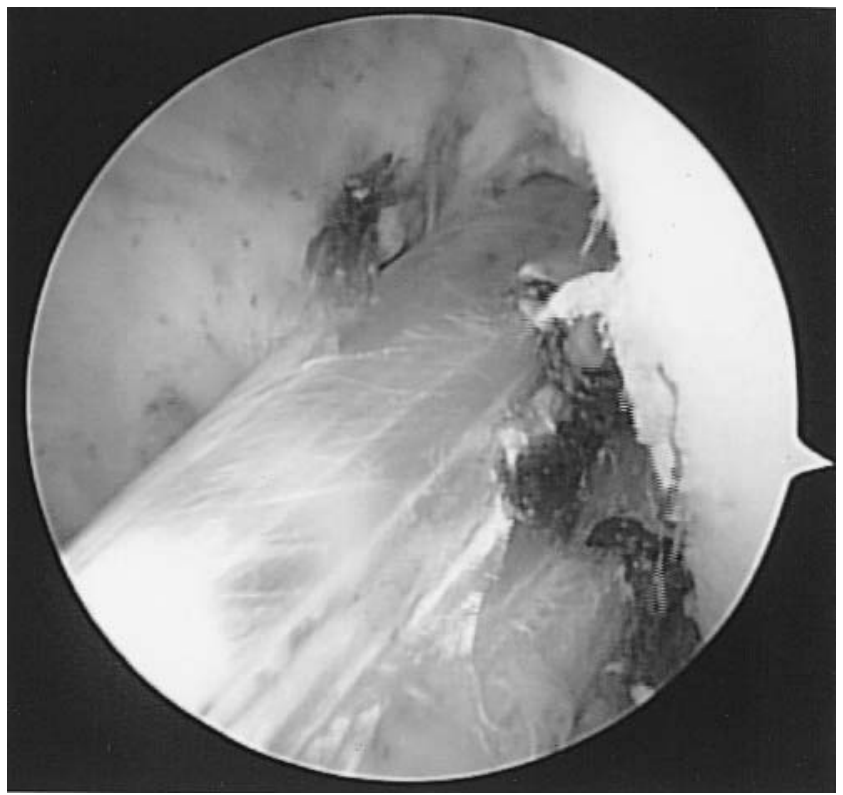

Fig. $4 b$

Arthroscopic views showing a) the position of two femoral sockets in the double-bundle reconstruction and b) grafts for the PL and AM bundles.

mm guide-wire was inserted into the selected position for the femoral attachment, and drilled through the femur to emerge on the lateral aspect of the thigh. After overdrilling using the Endobutton drill the diameter of which was 4.5 $\mathrm{mm}$, the femoral tunnel was created using the endoscopic cannulated drill. The length of the femoral tunnel was determined by the length of the graft and the distance between the exit foramina in the tibia and femur. The length of the femoral tunnel should be at least $21 \mathrm{~mm}$ (15 $\mathrm{mm}$ for the graft and $6 \mathrm{~mm}$ for the Endobutton flipping). The graft was passed through the tibial tunnel to the femoral tunnel and the Endobutton was 'flipped' and fixed to the lateral cortex of the femur. The excursion of the graft was checked. In all cases, the graft was pulled into the tibial tunnel with the knee approaching full extension. The total length of the excursion of the graft was $2.1 \pm 1.0 \mathrm{~mm}$ (0 to $5)$. A femoral notchplasty was performed when required. We applied a tension force of $50 \mathrm{~N}$ to the distal Endobutton tape of the graft and secured it with two staples with the knee at $90^{\circ}$ of flexion.

D group. For the double-bundle reconstruction, an elliptical tibial bone tunnel was created. A $2.0 \mathrm{~mm} \mathrm{~K}$-wire was inserted into the posterior half of the tibial attachment of the ACL and it was overdrilled with a cannulated reamer of the same diameter as that of the greatest diameter of the graft for the PL bundle. Using the parallel guide, we inserted the second K-wire $3 \mathrm{~mm}$ in front of the first (Fig. 3 ), and overdrilled it to the same diameter as the greatest diameter of the graft for the AM bundle. The tibial bone tunnel was chamfered, using a rasp, to make the tunnel elliptical in cross-section. The positions of the two femoral tunnels were at 11:00 and 9:30 o'clock (right) and 1:00 and 2:30 o'clock (left) with the same diameter as that of the proximal diameter of each graft for the AM and PL bundles (Fig. 4). Care was taken to leave a distance of at least $1 \mathrm{~mm}$ between the two tunnels to avoid overlapping. When both grafts had been passed through the tibial tunnel towards the femoral tunnels, the Endobuttons were 'flipped' and secured to the lateral cortex of the femur. The excursion of each graft was checked. In all cases, both grafts were pulled into the tibial tunnel with the knee approaching full extension. The total length of the excursion of the AM bundle was $1.6 \pm 1.5 \mathrm{~mm}$ (0 to 4$)$, and that of the PL bundle $2.2 \pm$ $1.4 \mathrm{~mm}$ (0 to 5). A femoral notchplasty was performed when required. A tension force of $25 \mathrm{~N}$ was applied equally to the distal Endobutton tape of each graft. They were fixed with double staples with the knee at $90^{\circ}$ of flexion.

The same post-operative regime was followed in all cases. Active quadriceps exercises were carried out as soon as possible. The knee was supported for two days in a brace, allowing $20^{\circ}$ of flexion. Range-of-movement exercises were initiated using a continuous passive motion device. Weight-bearing was allowed at seven days and jogging after three months. Strenuous sports activities were not allowed until nine months after surgery. There was no cases of infection, deep thrombosis, nerve or arterial injury, or any other complication.

Measurements of stability and proprioception were carried out before operation and at the final follow-up, more than two years after surgery. Anterior displacement of the tibia was measured using the KT-2000 knee arthrometer (MEDmetric, San Diego, California) with the knee at $20^{\circ}$ 
and $70^{\circ}$ of flexion. An anterior displacement force of $133 \mathrm{~N}$ was applied to the tibia of both the injured and normal knees, and the difference was recorded in millimetres. Tests of position sense were performed according to the method of Skinner, Barrack and Cook $^{17}$ using a Cybex II dynamometer (Cybext Co, Ronkonkoma, New York). The patient was asked to return the leg to a previous position. Inaccuracy was recorded as the difference between the perceived angle and the actual angle of flexion. The test was carried out ten times in each knee at low angles $\left(5^{\circ}\right.$ and $\left.25^{\circ}\right)$ and ten times at greater angles of flexion $\left(35^{\circ}\right.$ and $\left.80^{\circ}\right)$. The mean inaccuracy at low angles and greater angles was calculated separately for each knee. The final inaccuracy was expressed as the difference between the mean scores for the injured and the normal knee.

One of the authors (JI), who was blinded as to the group and details of each patient, performed all the tests of anterior stability and joint position sense.

Statistical analysis. The differences in the parameters between the two groups were analysed using the MannWhitney U and chi-square tests. The Wilcoxon signed-rank test was used for paired comparison. A p value of less than 0.05 was regarded as being statistically significant. All statistical analyses were carried out on Statview 5.0 (SAS Institite, Cary, North Carolina).

\section{Results}

Knee joint stability. The differences in anterior laxity, comparing the reconstructed with the normal knee, examined at $20^{\circ}$ of flexion in the $\mathrm{S}$ and $\mathrm{D}$ groups were (respectively) 5.4 $\pm 2.9 \mathrm{~mm}$ and $6.2 \pm 3.5 \mathrm{~mm}$ before operation and $1.2 \pm 2.5$ $\mathrm{mm}$ and $1.3 \pm 2.5 \mathrm{~mm}$ at final follow-up. The side-to-side differences of anterior laxity with the knee at $70^{\circ}$ of flexion in the $S$ and $D$ groups were $2.7 \pm 1.7 \mathrm{~mm}$ and $2.2 \pm 2.2 \mathrm{~mm}$ before operation and $1.5 \pm 2.0 \mathrm{~mm}$ and $1.2 \pm 1.6 \mathrm{~mm}$ at final follow-up. There was no statistically significant difference in outcome between the groups at either of these angles.

Joint position sense. The final inaccuracy at the low angles in the $\mathrm{S}$ and $\mathrm{D}$ groups, respectively, was $2.13 \pm 1.0^{\circ}$ and $1.91 \pm 0.8^{\circ}$ before operation and $0.75 \pm 1.4^{\circ}$ and $0.68 \pm 1.4^{\circ}$ at final follow-up. The final inaccuracy at the greater angles in the $S$ and $D$ groups was $2.18 \pm 1.0^{\circ}$ and $1.98 \pm 0.77^{\circ}$ before operation and $0.64 \pm 1.1^{\circ}$ and $0.67 \pm 1.1^{\circ}$ at the final follow-up. There was no statistically significant difference between the groups at any point in time after the surgery. Incidence of notchplasty. This was performed in 20 of 55 patients $(36.4 \%)$ in the $S$ group and in 10 of 53 patients $(18.9 \%)$ in the D group. There was a significant difference between the groups $(\mathrm{p}=0.04)$.

\section{Discussion}

Our study has shown no significant differences in the clinical outcome of between these two groups in terms of joint stability or position sense at different angles of flexion of the knee.
The normal ACL can be divided into two bundles, the $\mathrm{AM}$ and the PL. Amis and Dawkins ${ }^{1}$ further identified a separate intermediate bundle in the cadaver. The PL bundle is stretched in extension and the AM in flexion, which correlates with their contribution to the stability of the knee. Sakane et $\mathrm{al}^{3}{ }^{3}$ examining the forces in the human ACL found that the PL bundle took a greater load than the AM bundle, when the knee was flexed between zero and $45^{\circ}$. By contrast, the AM bundle remained relatively constant, not changing with the angle of flexion. At $90^{\circ}$ of flexion, the force taken by the AM bundle was greater than that taken by the PL bundle. Both bundles are under tension or relaxed at different angles of flexion, providing functional partial responsibility for the stability of the joint.

Reconstruction of the ACL using multistranded hamstring tendons is as popular as that using bone-patellar tendon-bone graft because it avoids morbidity at the donor site. With recent innovations in arthroscopic techniques in knee surgery a double-bundle reconstruction in which the $\mathrm{AM}$ and PL bundles are reconstructed separately to mimic more closely the normal anatomy of the ACL, has been advocated. Mae et $\mathrm{al}^{4}$ performed a cadaver study to compare the biomechanical differences between the single and double-bundle reconstruction and found better stability with the double reconstruction. Muneta et $\mathrm{al}^{6}$ reported the clinical outcome of double-bundle ACL reconstruction, two years after operation. Their results also showed greater stability with the double-bundle reconstruction. Hamada et $\mathrm{al}^{7}$ prospectively compared the clinical results of the singletunnel and the double-tunnel reconstruction. They found no significant difference between the two groups in terms of stability of the joint IKDC evaluation and muscle strength of the thigh. However, there have been few reports which have investigated the anterior stability of the knee in terms of the functional partial responsibility at the different angles of flexion of the knee. Our study has shown that the anterior stability in the double-bundle reconstruction at flexion angles of both $20^{\circ}$ and $70^{\circ}$ are not statistically significant compared with those in the single-bundle reconstruction. Further in vivo studies including histological and biomechanical evaluation are needed to explain these findings.

After Schultz et $\mathrm{al}^{8}$ described the existence of mechanoreceptors in the human cruciate ligament and suggested their proprioceptive role, others have reported similar findings. It is now accepted that the normal ACL is extensively innervated by mechanoreceptors with an important afferent function.

Iwasa et $\mathrm{al}^{16}$ investigated the improvement of the proprioceptive function in the knee after reconstruction of the ACL. They found that 30 of 38 patients improved their position sense after reconstruction after an interval of 18 months suggesting perhaps that mechanoreceptors had regenerated in the new ACL. Some reinnervation of mechanoreceptors has been demonstrated histologically in the reconstructed ACL in experimental models using sheep, goats, and dogs. Goertzen et $\mathrm{al}^{12}$ reported that mechano- 
receptors were present beneath the synovium covering the reconstructed ACL at six and 12 months after surgery but they were unable to investigate their function. Ochi et $\mathrm{al}^{10}$ found detectable somatosensory evoked potentials by electrical and mechanical stimulation in the reconstructed ACL with autogenous hamstring tendons suggesting that sensory innervation can occur in the reconstructed ACL.

Since the cross-sectional and surface areas of the doublebundle graft will be greater than those of the single-bundle reconstruction, the post-operative proprioceptive function in the double-bundle reconstruction should be better, but this has not been the case.

As Hamada et $\mathrm{al}^{7}$ have reported, there are several theoretical advantages of the double-bundle ACL reconstruction. It should have an advantage in graft-bone healing because the graft-bone contact area is greater than that in the single-bundle reconstruction. Furthermore, the incidence of the requirement for notchplasty is lower than that in the single-bundle reconstruction.

In our study, we could not demonstrate an advantage of double-bundle reconstruction in terms of stability of the joint or proprioceptive function over the single-bundle reconstruction and we suggest that there is no need for double-bundle reconstruction.

No benefits in any form have been received or will be received from any commercial party related directly or indirectly to the subject of this article.

\section{References}

1. Amis AA, Dawkins PC. Functional anatomy of the anterior cruciate ligament: fibre bundle actions related to ligament replacements and injuries. J Bone Joint Surg [Br] 1991;73-B:260-7.
2. Dodds JA, Arnoczky SP. Anatomy of the anterior cruciate ligament: a blueprint for repair and reconstruction. Arthroscopy 1994;10:132-9.

3. Sakane M, Fox RJ, Woo SL-Y, et al. In situ force in the anterior cruciate ligament and its bundle in response to anterior tibial loads. J Orthop Res 1997;15:285-93.

4. Mae T, Shino K, Miyama T, et al. Single- versus two-femoral socket anterior cruciate ligament reconstruction technique: biomechanical analysis using a robotic simulator. Arthroscopy 2001;17:708-16.

5. Yagi M, Wong EK, Kanamori A, et al. Biomechanical analysis of an anatomic anterior cruciate ligament reconstruction. Am J Sports Med 2002;30:660-6.

6. Muneta T, Sekiya I, Yanagishita K, et al. Two-bundle reconstruction of the anterior cruciate ligament using semitendinosus tendon with Endobutton: operative technique and preliminary results. Arthroscopy 1999:15:618-24.

7. Hamada M, Shino K, Horibe $\mathbf{S}$, et al. Single- versus Bi-socket anterior cruciate ligament reconstruction using autogenous multiple-stranded hamstring tendons with Endobutton femoral fixation: a prospective study. Arthroscopy 2001;17:801-7.

8. Schultz RA, Miller DC, Kerr CS, Micheli L. Mechanoreceptors in human cruciate ligaments: a histologic study. J Bone Joint Surg [Am] 1984;66-A:1072-6.

9. Schutte MJ, Dabezies El, Zimmy ML, Happel LT. Neural anatomy of the human anterior cruciate ligament. J Bone Joint Surg [Am] 1987;69-A:243-7.

10. Ochi M, Iwasa J, Uchio Y, Adachi N, Sumen Y. The regeneration of sensory neurons in the reconstruction of the anterior cruciate ligament. $J$ Bone Joint Surg $[\mathrm{Br}]$ 1999:81-B:902-6.

11. Adachi N, Ochi M, Uchio Y, et al. Mechanoreceptors in the anterior cruciate ligament contribute to the joint position sense. Acta Orthop Scand 2002;73:330-4.

12. Goertzen M, Gruber J, Dellmann A, Clahsen H, Schulitz KP. Neurohistological findings after experimental anterior cruciate ligament allograft transplantation. Arch Orthop Trauma Surg 1992;111:126-9.

13. Denti M, Monteleone M, Berardi A, Panni AS. Anterior cruciate ligament mechanoreceptors. Clin Orthop 1994;308:29-32.

14. Barrack RL, Lund PJ, Munn BG, Wink C, Happel L. Evidence of reinnervation of free patellar tendon autograft used for anterior cruciate ligament reconstruction. Am J Sports Med 1997:25:196-202.

15. Shimizu T, Takahashi T, Wada Y, et al. Regeneration process of mechanoreceptors in the reconstructed anterior cruciate ligament. Arch Orthop Trauma Surg 1999;119:405-9.

16. Iwasa J, Ochi $\mathbf{M}$, Adachi $\mathbf{N}$, et al. Proprioceptive improvement in knees with anterior cruciate ligament reconstruction. Clin Orthop 2000;381:168-76.

17. Skinner HB, Barrack RL, Cook SD. Age-related decline in proprioception. Clin Orthop 1984;184:208-11. 